\title{
FROM THE KINETIC ENERGY RECOVERY SYSTEM TO THE THERMO-HYDRAULIC HYBRID MOTOR VEHICLE
}

\author{
Dr. Ing. Corneliu CRISTESCU, Ph.D., Senior Researcher \\ Dr. Ing. Petrin DRUMEA, Ph.D., Senior Researcher \\ Dr. Ing. Dragos Ion GUTA, Ph.D., Junior Researcher \\ Ing. Catalin DUMITRESCU, Ph.D. St., Junior Researcher
}

Hydraulics and Pneumatics Research Institute INOE 2000-IHP, Bucharest, ROMANIA

\begin{abstract}
The paper presents some theoretical and experimental results obtained by the Hydraulics and Pneumatics Research Institute INOE 2000-IHP with its partners, regarding the creating of one hydraulic system able to recovering the kinetic energy of the motor vehicles, in the braking phases, and use this recovered energy in the starting and accelerating phases. Also, in the article is presented a testing stand, which was especially designed for testing the hydraulic system for recovery the kinetic energy. Through mounting of the kinetic energy recovering hydraulic system, on one motor vehicle, this vehicle became a thermo-hydraulic hybrid vehicle. Therefore, the dynamic behavior was analyzed for the whole hybrid motor vehicle, which includes the energy recovery system. The theoretical and experimental results demonstrate the possible performances of the hybrid vehicle and that the kinetic energy recovery hydraulic systems are good means to increase energy efficiency of the road motor vehicles and to decrease of the fuel consumption.
\end{abstract}

KEYWORDS: Regenerative Driving Systems, Energy Recovering Systems, Hybrid Motor Vehicles

\section{INTRODUCTION}

As it known, in the braking phase of the motor vehicle, the kinetic energy accumulated in the accelerating phase is converted in the thermal energy, which is, normally and irremediable, wasted in atmosphere, which has a negative impact over the environment and fossil fuels resource. To reduce the fuel consumption and to limit the gas emission, have appeared the energy recovery systems at the braking of motor vehicle, which used different technical solutions for capturing, storing and reusing of the this energy [1]. Romanian solution is only one of them [2]. For the conceiving of the recovery system and, also, for the testing stand, was used the new mechatronics technology, the single technology which is able to manage this very complex system. The kinetic energy recovery system contains the next main subsystem: a hydro-mechanic subsystem, an electronics subsystem, an informatics subsystem and, also, a control subsystem [3] The propulsion systems that have in their composition, in addition to a conventional internal combustion engine, at least one capable of providing torque to the wheels traction vehicles and recover some of the kinetic energy, are known as hybrid regenerative systems [4], [5].

This is a new automotive technology, which successes a substantial reducing of fuel consumption and gas emissions. Manny companies from the world have developed this kind of propulsion systems, with the main objectives to capture and recovey the kinetic energy at the braking of motor vehicles for reducing the fuel consumption and the environment pollution. In the world, were 
SCIENTIFIC PROCEEDINGS 2011, Faculty of Mechanical Engineering, STU in Bratislava .

Vol. 19. 2011, pp. 80-86, DOI: 10.2478/v10228-011-0014-Z

created many technical solutions for hybrid propulsion systems that can recovery the kinetic energy from the motor vehicles. But, the most widespread systems are the thermo-electric hybrid systems and the thermo-hydraulic hybrid systems.

\section{THE ROMANIAN KINETIC ENERGY RECOVERY SYSTEM}

In order to achieve the kinetic energy recovery system, the adopted technical solution was that of kinetic energy recovery by hydraulic means. In the braking phases, the kinetic energy of the motor vehicle is converted into hydrostatic energy, which is stored at high pressure in hydropneumatic accumulators by one hydraulic machine, which works as a pump or as a motor. In the acceleration/start-up stages, the hydrostatic energy, stored in hydro-pneumatic accumulators in the braking phases, is converted back into mechanical energy by the hydraulic machine that is working now as a hydraulic motor and which will generate the acceleration of the motor vehicle [3].

\section{CONSTRUCTIVE CONFIGURATION AND IMPLEMENTATION OF ENERGY RECOVERY SYSTEM ON MOTOR VEHICLES}

Constructive and functional concept of developing and implementing a system for braking energy recovery is shown, in schematically, in Fig. 1, which presents a conceptual model of construction and installation/implementation of the kinetic energy recovery system on a motor vehicle. The energy recovery system consists, in essence, of a hydro-mechanical module which includes a variable displacement hydraulic machine, that can operate both in pump mode, during braking, and in engine mode, during start-up/acceleration of the motor vehicle. The hydro-mechanic module is connected in a parallel line with the existing thermo-mechanic drive line of the vehicle, in order to capture and store the kinetic energy of vehicle, in the braking phase [2].

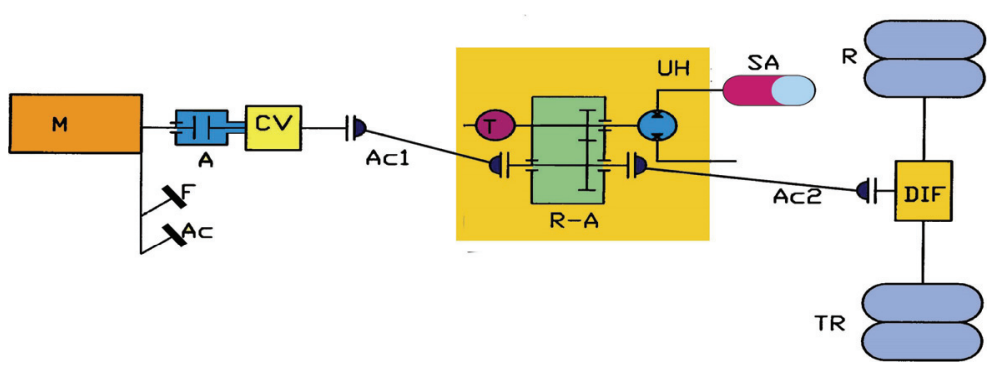

Fig. 1 A conceptual model of construction and installation/implementation of the recovery system on motor vehicles

The implementation/installation of the energy recovery system can be done on motor vehicles that have a long Cardan axle between the gearbox CV and the differential mechanism DIF, by replacing it with two shorter axles. Mechanical connection between the Cardan axles Ac1 and Ac2 and the recovery system R-A is permanent and is achieved through a mechanical transmission, which adapts the rotational speed of the Cardan axle to the operating rotational speed of the hydraulic machine/unit UH in the system. Depending on the specific conditions provided by the motor vehicle on which the recovery system is installed, the coupling outlet and mechanical 
SCIENTIFIC PROCEEDINGS 2011, Faculty of Mechanical Engineering, STU in Bratislava .

Vol. 19. 2011, pp. 80-86, DOI: 10.2478/v10228-011-0014-Z

transmission can be placed at the end of the Cardan axle Ac1 close to the gearbox, at the end of the Cardan axle close to the rear drive train TR, or between the gearbox CV and the drive train TR, by splitting the Cardan axle. The hydraulic unit is a hydraulic machine of variable displacement/geometric volume, which can vary between 0 and a maximum value $\left(\mathrm{V}_{\mathrm{g}}=\mathrm{max}\right)$. Axial piston hydraulic unit can be removed from the zero displacement position, only when the vehicle goes forward. When it goes into reverse, the displacement of the unit remains zero $(\mathrm{Vg}=0)$. Recovery system, also, comprises the hydraulic station to achieve hydraulic circuits, as well as the transducers required for monitoring and automation of braking and acceleration processes.

The hydraulic machine, which is driven by a mechanical transmission, is controlled by an electric and electronic control subsystem, which performs also the interfacing with the braking and acceleration systems of the basic motor vehicle, operation being controlled through a processor, which provides the information support specific to mechatronic systems [3].

The implementation/mounting of the kinetic energy recovery hydraulic system, on the motor vehicle with clasical thermo-mechanical propulsion system, leads to transformation of the motor vehicle into a thermo-hydraulic hybrid vehicle with a hibrid propulsion system.

\section{GLOBAL SYSTEMIC MODEL OF THE HYBRID VEHICLES WITH KINETIC ENERGY RECOVERY SYSTEM}

According to the theory of automatic systems, the global systemic model of the hybrid vehicle with kinetic energy recovery system is shown in Fig. 2.

\begin{tabular}{|c|c|c|c|c|}
\hline \multirow{2}{*}{$\begin{array}{c}\text { VEHICLE DRIVETRAIN } \\
\text { - VDR - } \\
\text { (REAR AXLE) }\end{array}$} & bracking & \multirow{2}{*}{$\begin{array}{c}\text { ENERGY RECOVERY } \\
\text { SYSTEM } \\
\text { - ERS - } \\
\text { (HYDRAULIC SYSTEM) }\end{array}$} & bracking & \multirow{2}{*}{$\begin{array}{c}\text { ENERGY STORAGE } \\
\text { SYSTEM } \\
\text { - ESS - } \\
\text { (PNEUMO - HYDRAULIC } \\
\text { ACCUMULATORS) }\end{array}$} \\
\hline & 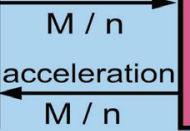 & & $\begin{array}{c}\frac{p / Q}{\text { acceleration }} \\
\frac{p / Q}{p}\end{array}$ & \\
\hline
\end{tabular}

Fig. 2 Global systemic model of a motor vehicle equipped with a kinetic energy recovery system

During the braking stage, the recovery system ESR captures, from the drive train VDR, the vehicle's kinetic energy (with mechanical parameters: torque/moment $M$ and rotational speed $n$ ), converts it into hydrostatic energy (with hydraulic parameters: pressure $p$ and flow $Q$ and stores it inside the storage subsystem ESS. During the acceleration or start-up phases, the hydrostatic energy (with hydraulic parameters: pressure $p$ and flow $Q$ is transmitted to the recovery system SR which converts it into mechanical energy (with mechanical parameters: torque $M$ and rotational speed $n$ ), and uses it to add torque/moment to the propulsion and drive train of the vehicle, for acceleration or start-up, as appropriate. The general systemic model of interfacing and inter conditioning of the energy recovery system with the all systems, that command and control motor vehicle movement (braking and acceleration systems), is shown roughly in Fig. 3. 


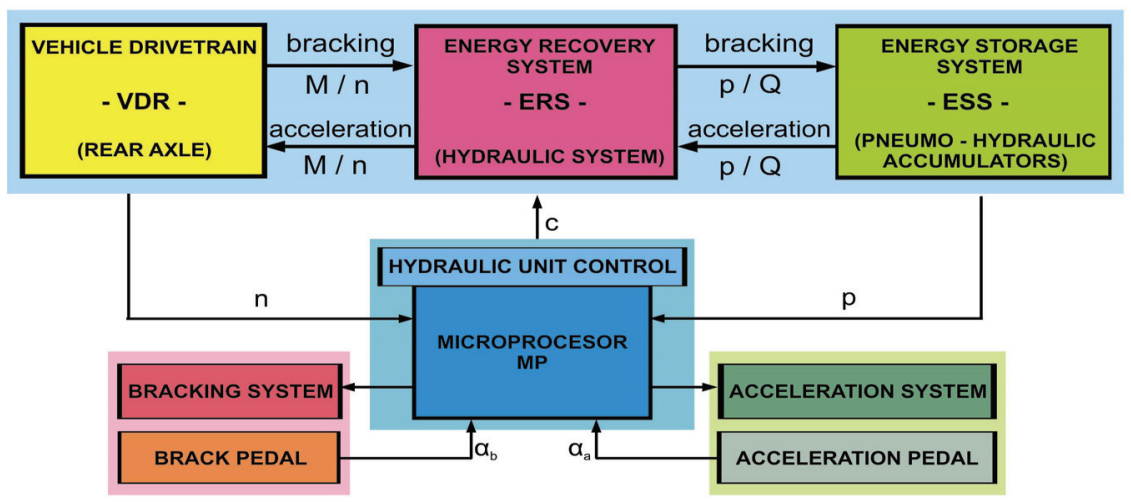

Fig. 3 General systemic model of the command and control system

The microprocessor MP receives information on the braking or acceleration command, rotational speed, pressure inside the storage system, and manages the entire process through commands sent to the recovery system and to the conventional braking or acceleration systems.

The automatic adjustment system of the motor vehicle hybrid propulsion system has an adjustment system which achieves proportionality between the stroke of the brake pedal and the stroke of the acceleration pedal, on slowing down, respectively, on starting-up the motor vehicle.

\section{MECHATRONICS STRUCTURE OF THE HYBRID MOTOR VEHICLES WITH KINETIC ENERGY RECOVERY SYSTEM}

The hybrid motor vehicles with kinetic energy recovery system have a typical mechatronics structure [2], [3], consisting of the next three main subsystems:

1. mechanical-hydraulic subsystem, which consists of hydro-mechanical module, hydraulic station, battery of hydro pneumatic accumulators,

2. electronic drive and control subsystem, which consists of all electric, electronic, sensors and automation components that ensure system operation, including the drive and control panel;

3. informatics/ computer subsystem for process control, consisting of user licensed purchased software or software specifically designed and dedicated to the proper functioning.

\section{THE CONCEPTUAL MODEL OF THE ROMANIAN KINETIC ENERGY RECOVERY HYDRAULIC SYSTEM}

The energy recovering hydraulic system was designed to be implemented on one Romanian motor vehicle, well-known as ARO 243 type, which has a 4x4 driving system, Fig. 4. 


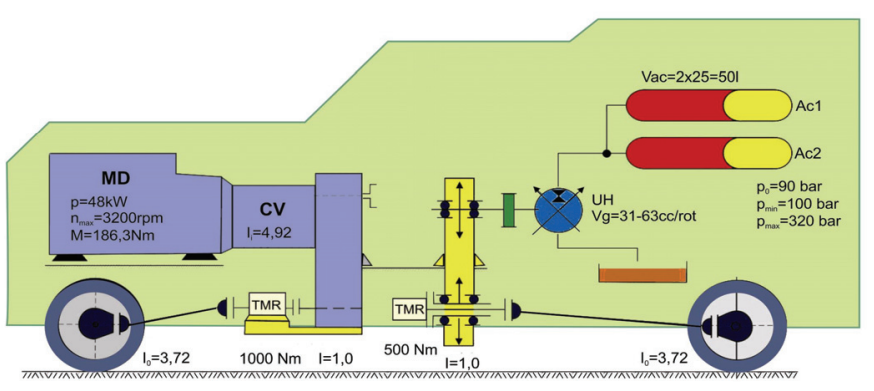

Fig. 4 Conceptual model of the Romanian kinetic energy recovery hydraulic system

The Fig. 4 distinguishes the Diesel engine MD, the gearbox CV and the gear transmission to the front wheels, through one torque and rotation transducer (TMR) and one cardan axle. Also, the figure distinguishes the mechanical transmission to the hydraulic machine/unity $\mathrm{UH}$, the low pressure thanks LT and the height pressure stored system compounds of the two hydraulic and pneumatic accumulators AC1 and AC2. The hydraulic power driving is transmitted to the breech wheels through the one torque and rotation transducer (TMR) and one cardan axle. The hydraulic machine can be connected, in parallel, anywhere in the driveline, but, generally, it is mounted in the driveshaft, between gearbox and differential mechanism. The main part of the energy recovery system is the hydraulic machine with variable geometrical volume. The hydraulic machine is acted through one transmition, being assisted by an electro-hydraulic system, interfaced with the braking and acceleration subsystems of the vehicle.

\section{THE MAIN PHYSICAL MODULES OF THE KINETIC ENERGY RECOVERY HYDRAULIC SYSTEM}

The main module of the kinetic energy recovery system, are presented in the Fig. 5:

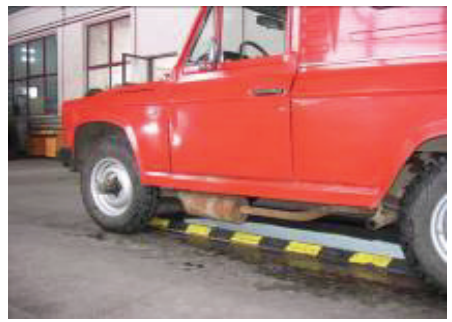

(a) The motor vehicle ARO-243

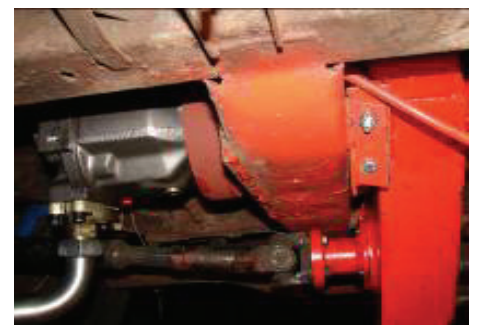

(b) The hydro-mechanical module 


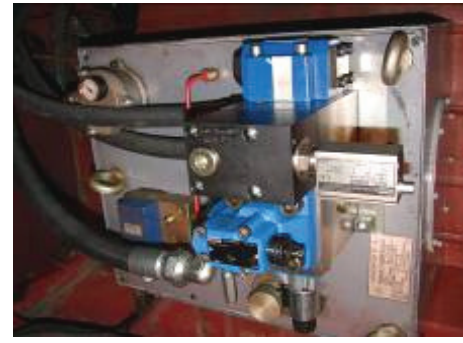

(c) The hydraulic station

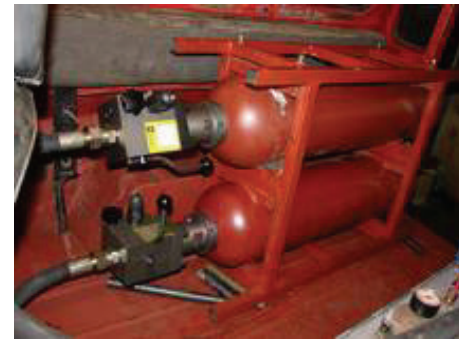

(d) The accumulators battery

Fig. 5 The main modules of the kinetic energy recovery system

\section{SOME THEORETICAL RESULTS REGARDING THE DYNAMIC BEHAVIOR OF THE HYBRID MOTOR VEHICLE}

In order to know the dynamic behavior of the hybrid motor vehicle, were elaborated the mathematical models and the simulation programs for the starting and braking phase and had been obtained a lot of variation diagrams. For exemple, the diagram for the energy efficiency at the hydraulic propulsion is shown in Fig. 6 and for braking energy recovery coefficient is in Fig. 7.

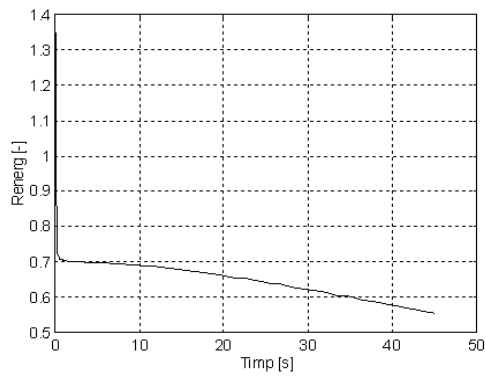

Fig. 6 Variation of energy efficiency at the hydraulic propulsion

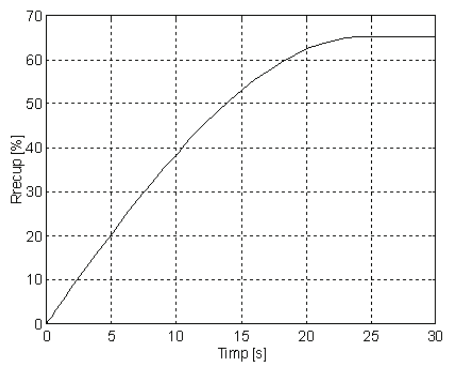

Fig. 7 Variation of braking energy recovery coefficient

\section{THE TESTING STAND OF THE KINETIC ENERGY RECOVERY SYSTEM}

For testing of the functionality of the components of the recovery system, was designed and manufactured a test stand which is presented in the Fig. 8. The stand allows to obtain a lot of experimental graphics. For exemple, in Fig. 9, is presented a diagrame with the variation of the torque at the drive shaft, delivered from thermal system, hibrid system and the hydraulic machine. 


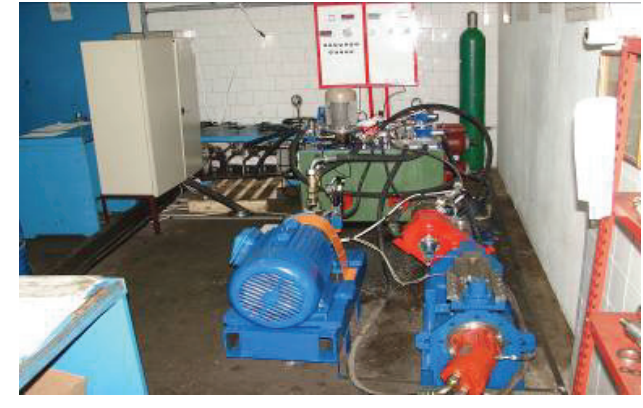

Fig. 8 The testing stand of the energy recovery system

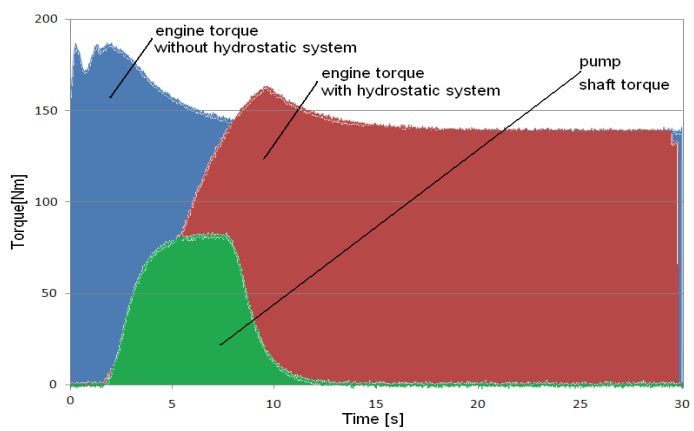

Fig. 9 The torque variation at the drive shaft

\section{CONCLUSIONS}

The paper made a summary presentation of one Romanian hydraulic system for kinetic energy recovery at motor vehicles and are given some theoretical and experimental result.

The paper shows the possibility to design, manufacture and implementing the kinetic energy recovery hydraulic systems on motor vehicles that, through mounting of the recovery system, transforms it in hybrid motor vehicles, with thermo-hydraulic propulsion hybrid systems.

The theoretical result demonstrates the possible performances of the hybrid vehicles, the hydraulic systems being good solutions to increase energy efficiency of the road vehicles.

The adopted technical solution allows extrapolation to different sizes of vehicles, for medium and heavy vehicles, and can be mounted on vehicles both new and old motor vehicles.

\section{REFERENCES}

[1] BOSCH REXROTH GROUP, Hydraulic Hybrids: System HRB, 2010, Available from: http://www.boschrexroth.com/country_units/america/united_states/sub websites/brus_brh_m/en/markets_applications_jg/a_downloads/ra98310_2010-08.pdf

[2] CRISTESCU, C.: The recovery of kinetic energy at braking of motor vehicle, Publishing House AGIR, Bucharest, Romania, 2008, ISBN 978-973-720-219-2.

[3] CRISTESCU, C., DRUMEA, P., ION GUTA, D. D., DUMiTRESCU, C. CHIRITA, C.: Hydraulic system for recovery kinetic energy at the braking of the medium and heavy motor vehicles. In; Proceedings of The 21th International Conference on Hydraulics and Pneumatics, Ostrava, Czech republic, 1-3 June, 2011, ISBN 978-80-248-2430-7

[4] PARKER HANNIFIN (2010). Fluid power: Saving Energy with Efficient Fluid Power /Hydraulic Hybrid Drive System Available from: http://www.parker.com, and http://www.mobilehydraulictips.com/tag/parker-hannifin/page/2/

[5] PERMO-DRIVE (2009). What is the Regenerative Drive System (RDS $\left.{ }^{T M}\right)$ ? Available from: www.permodrive.com and http://www.permodrive.com/tech/index.htm 